\title{
Silicon Photonic MEMS: Exploiting Mechanics at the Nanoscale to Enhance Photonic Integrated Circuits
}

\author{
Niels Quack ${ }^{1}$, Hamed Sattari ${ }^{1}$, Alain Y. Takabayashi ${ }^{1}$, Yu Zhang ${ }^{1}$, Pierre Edinger ${ }^{2}$, Carlos Errando- \\ Herranz $^{2}$, Kristinn B. Gylfason ${ }^{2}$, Xiaojing Wang ${ }^{2}$, Frank Niklaus ${ }^{2}$, Moises A. Jezzini ${ }^{3}$, How Yuan Hwang ${ }^{3}$, \\ Peter O'Brien ${ }^{3}$, Marco A. G. Porcel ${ }^{4}$, Cristina Lerma Arce ${ }^{5}$, Saurav Kumar ${ }^{5}$, Banafsheh Abasahl ${ }^{6}$, Peter \\ Verheyen $^{6}$ and Wim Bogaerts ${ }^{6}$ \\ ${ }^{1}$ École Polytechnique Fédérale de Lausanne (EPFL), CH-1015 Lausanne, Switzerland \\ ${ }^{2}$ KTH Royal Institute of Technology, Malvinas väg 10, SE-100 44 Stockholm, Sweden \\ ${ }^{3}$ Tyndall National Institute, Lee Maltings Complex Dyke Parade, T12 R5CP Cork, Ireland \\ ${ }^{4}$ VLC Photonics S.L., Ed. 9B, D2, UPV, Camino de vera sn, 46022 Valencia, Spain \\ ${ }^{5}$ Commscope Connectivity Belgium, Diestsesteenweg 692, 3010 Kessel LO, Belgium \\ ${ }^{6}$ Ghent University - IMEC, Department of Information Technology, Photonics Research Group \\ Technologiepark-Zwijnaarde 15, 9052 Gent, Belgium \\ niels.quack@epfl.ch
}

\begin{abstract}
With the maturing and the increasing complexity of Silicon Photonics technology, novel avenues are pursued to reduce power consumption and to provide enhanced functionality: exploiting mechanical movement in advanced Silicon Photonic Integrated Circuits provides a promising path to access a strong modulation of the effective index and to low power consumption by employing mechanically stable and thus non-volatile states. In this paper, we will discuss recent achievements in the development of MEMS enabled systems in Silicon Photonics and outline the roadmap towards reconfigurable general Photonic Integrated Circuits.

OCIS codes: (250.5300) Photonic Integrated Circuits; (230.4685) Optical Microelectromechanical Devices.
\end{abstract}

\section{Introduction}

Over the past two decades, Silicon Photonics has emerged from exploratory research and developed into commercial offerings of Silicon Photonic based products manufactured in volume. The tremendous success of Silicon Photonics as technology of choice for integrated photonics has been underpinned by the development of mature and standardized silicon photonic platform offerings. While current silicon photonics technology addresses existing markets such as optical interconnects in datacenters, the technology platforms offer various opportunities for enhancement, both in performance and applications, thereby opening avenues to address new markets: promising avenues include, among others, the introduction of additional materials for light sources, high-speed modulators or ultra-low loss waveguides.

Nevertheless, such modifications will require major changes in the fabrication process flows. A promising way to enhance functionality without significant disruption of established process flows is enabling mechanical movement of silicon waveguides by locally removing the cladding oxide surrounding the silicon waveguides, effectively 'releasing' the silicon structures. While photonic designs need to be adapted to account for the modified waveguide cladding, suitable release process steps can be performed at the back-end-of-line with virtually zero change to the standard platform technology. Introducing mechanical movement to Photonic Integrated Circuits (PIC) provides an excellent opportunity to enhance performance by virtue of a strong modulation of the effective index, and by the possibility to include mechanically stable states, which drastically reduces static power consumption.

\section{Silicon Photonic MEMS}

Among the material platforms for photonic integrated circuits, silicon photonics is particularly attractive for including mechanical components, due to the excellent mechanical material properties and the mature MEMS technology basis available for silicon devices. In order to perform elementary functions, such as switching, phase shifting or variable attenuation, displacement of waveguides in the range of several hundreds of nanometers is sufficient to achieve full $2 \pi$ phase shift [1], or to fully (de-)couple optical signals in adjacent waveguides, as required for optical switching [2]. With adequate design, electrostatic actuators can achieve the required movements at low voltages and with low power consumption.

In order to include mechanically movable structures in photonic integrated circuits, various strategies have been implemented, such as single-layer movable waveguide structures [3, 4], or displacement of movable structures in multi-layer arrangements $[5,6]$. While multi-layer structures provide additional degrees of freedom in design, they 
require surface micromachining fabrication processes that typically involve deposition and annealing steps at temperatures between $500^{\circ} \mathrm{C}$ and $900^{\circ} \mathrm{C}$. Such high temperatures are typically not compatible with active components (photodiodes, modulators), making this technique less suitable for inclusion in today's most advanced silicon photonics foundry platforms. In contrast, the approach based on mechanical movement of silicon structures fabricated in the waveguide layers of the SOI device layer is fully compatible with standard silicon photonics.

\section{MEMS in Standard Silicon Photonics Technology}

In order to keep and leverage the existing optimized optical functions available in advanced Silicon Photonic foundry platforms, such as enhanced grating couplers, photodetectors, electrical interconnect wiring, modulators, etc., we pursue in the scope of the MORPHIC project [7] a 'zero photonics process change' approach, enabling MEMS actuated structures by post-processing steps only. We demonstrate the MEMS release process consisting of a window opening and sacrificial layer etch [8] on IMEC's ISIPP50G platform [9], with all process steps $200 \mathrm{~mm}$ wafer compatible (Fig. 1a). Examples of freestanding photonic devices such as waveguide couplers and ring resonators fabricated using this approach are shown in Fig. $1 \mathrm{~b}$ and 1c, respectively. The key functionalities that we aim to implement as photonic MEMS are low power optical phase shifters [10] and tunable $2 \times 2$ (directional) couplers. Furthermore, we are developing hermetic packaging for long-term reliability and stable performance of the photonic MEMS.
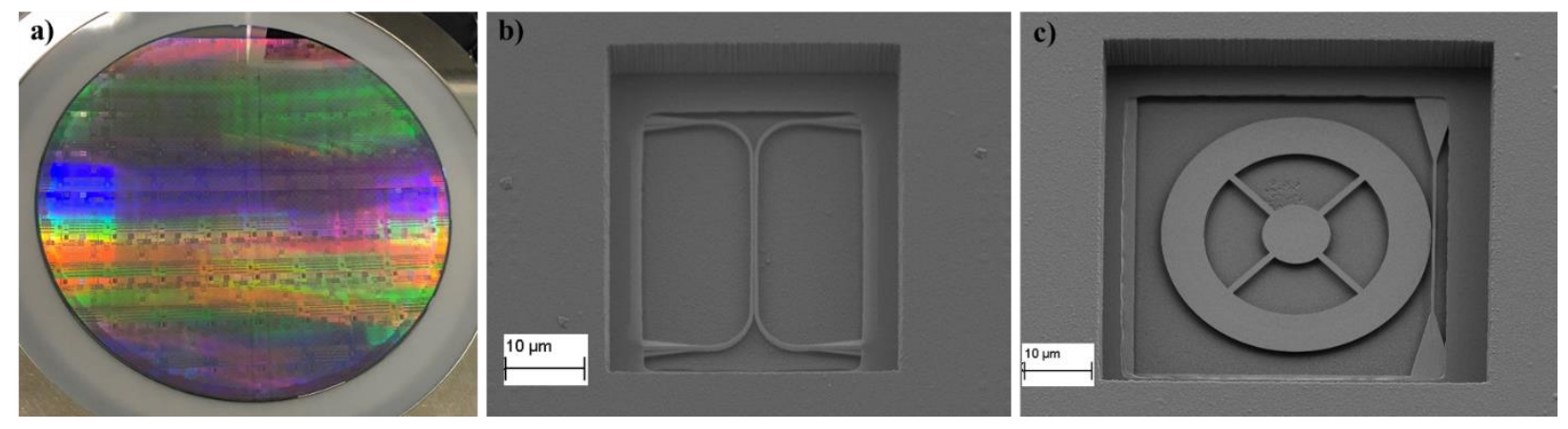

Figure 1: a) Photograph of a processed Silicon Photonics $200 \mathrm{~mm}$-Wafer and SEM recordings showing examples of released Silicon Photonic MEMS structures consisting of b) freestanding waveguides forming a directional coupler and c) a freestanding waveguide coupled to a microdisk resonator.

\section{Mechanically Reconfigurable Photonic Integrated Circuits}

The large-scale integration of such individual MEMS tunable building blocks in a standard silicon photonics platform is especially suitable for the emerging concept of reconfigurable (programmable) photonic circuits [11, 12], which rely heavily on the use of phase shifters and tunable couplers. A wafer-scale silicon photonics platform with MEMS could enable reconfigurable photonics and offer a convenient platform for rapid prototyping of new photonic concepts, as conceptually depicted in Figure 2.

\section{Summary}

We demonstrate silicon photonic MEMS building blocks by a post-processing release step on IMEC's standard advanced silicon photonics platform. Large-scale integration of such elementary MEMS-enabled unit cells, including phase shifters and tunable couplers, in silicon photonic integrated circuits, can lay the foundation for building photonic-networks on-chip that can be reprogrammed on-demand by reconfiguring the individual photonic MEMS building blocks. With advanced packaging [13] and on-demand programming of large-scale silicon photonic MEMS circuits to fit the customer's requirements, such field-programmable photonic integrated circuits (FP-PICs) may well re-shape the development cycles of photonic integrated systems.

\section{Acknowledgements}

This project has received funding from the European Union's Horizon 2020 research and innovation programme under grant agreement No 780283 (MORPHIC - www.h2020morphic.eu). N. Quack acknowledges funding from the Swiss National Science Foundation (SNSF) under grant No. 157566, and H. Sattari from the Hasler Foundation under grant No. 17008. 


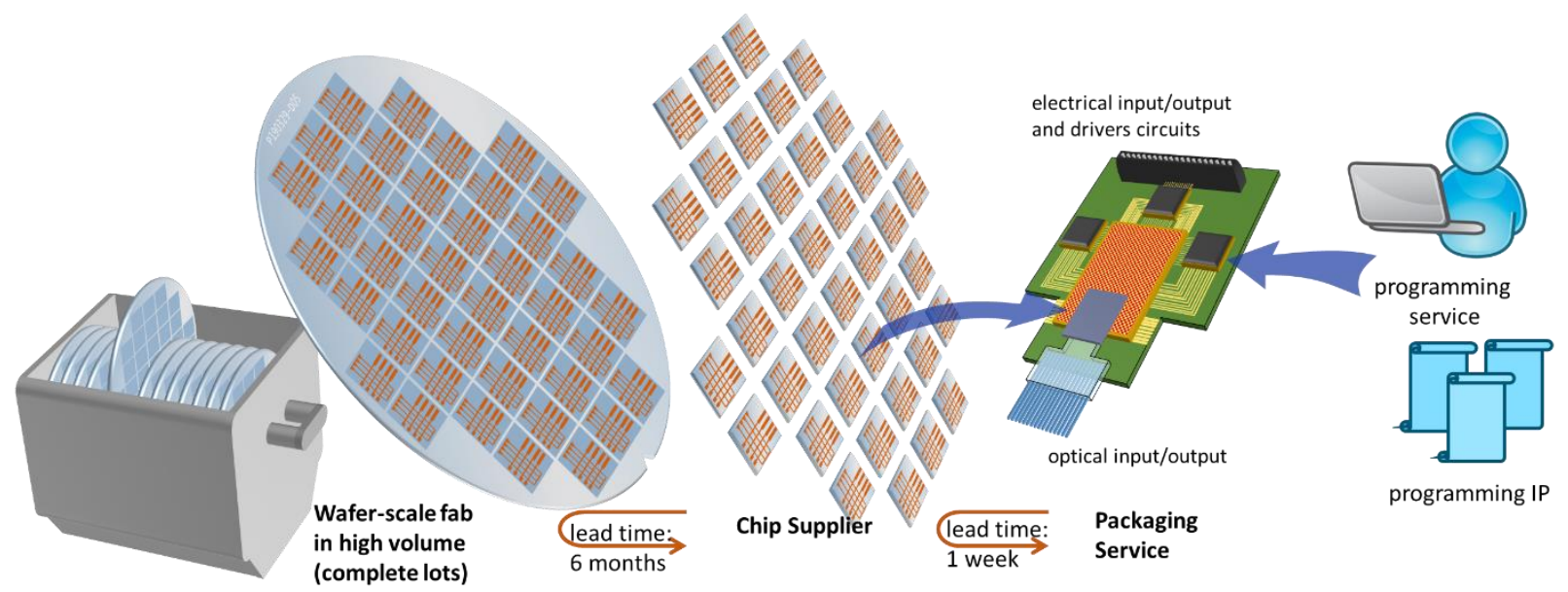

Figure 2: Conceptual representation of an ecosystem built on programmable photonics: MEMS-reconfigurable photonic integrated circuits can be programmed on-demand for customers, providing customized photonic integrated circuits in shortened turn-around times.

\section{References}

[1] K. Van Acoleyen, J. Roels, P. Mechet, T. Claes, D. Van Thourhout, and R. Baets, "Ultracompact Phase Modulator Based on a Cascade of NEMS-Operated Slot Waveguides Fabricated in Silicon-on-Insulator," IEEE Photonics Journal, 4, 3, 2012.

[2] S. Han, T.J. Seok, N. Quack, B.-W. Yoo, and M.C. Wu, "Large-scale silicon photonic switches with movable directional couplers," Optica, 2, 4, 2015.

[3] C. Errando-Herranz, N. Le Thomas, K.B. Gylfason, "Low-power optical beam steering by microelectromechanical waveguide gratings," arXiv: 1809.04483 [physics. app-ph], 2018.

[4] S. Abe and K. Hane, "Variable-Gap Silicon Photonic Waveguide Coupler Switch With a Nanolatch Mechanism," IEEE Photonics Technology Letters, 25, 7, 675-677, 2013.

[5] T. J. Seok, N. Quack, S. Han, R. S. Muller and M. C. Wu, "Large-scale broadband digital silicon photonic switches with vertical adiabatic couplers," Optica, 3, 1, 2016.

[6] S. Han, T. J. Seok, K. Yu, N. Quack, R. S. Muller and M. C. Wu, "Large-Scale Polarization-Insensitive Silicon Photonic MEMS Switches,” Journal of Lightwave Technology, 36,10, 2018.

[7] MORPHIC project website https://h2020morphic.eu/

[8] J. Jacobs, T. Graziosi, M. Kiss, S. Han, T. J. Seok, M. C. Wu, N. Quack, "Die Level Release of Silicon Photonic MEMS," in International Conference on Optical MEMS and Nanophotonics (IEEE OMN), 2016.

[9] P. P. Absil, P. Verheyen, P. De Heyn, M. Pantouvaki, G. Lepage, J. De Coster, and J. Van Campenhout, "Silicon photonics integrated circuits: a manufacturing platform for high density, low power optical I/O's," Optics Express, 23, 7, 9369-9378, 2015.

[10] P. Edinger, C. Errando-Herranz, and K.B. Gylfason, "Low-Loss MEMS Phase Shifter for Large Scale Reconfigurable Silicon Photonics," accepted for publication in International Conference on Micro Electro Mechanical Systems (IEEE MEMS), 2019.

[11] D. Perez, I. Gasulla, and J. Capmany, "Towards programmable microwave photonic processors," Journal of Lightwave Technology, vol. 36, no. 2, pp. 519-532, 2018.

[12] J. Carolan, et al., "Universal linear optics," Science, 349, 6249, 711-716, 2015.

[13] H. Y. Hwang et al., "Flip Chip Packaging of Digital Silicon Photonics MEMS Switch for Cloud Computing and Data Centre," IEEE Photonics Journal, 9, 3, 2017. 